\title{
Photons from heavy-ion collisions at Fermi velocity
}

\author{
Che Ming Ko \\ Center for Theoretical Physics, Physics Department and Cyclotron Institute, Texas A\&M University, \\ College Station, Texas 77843 \\ Jörg Aichelin \\ Institut für Theoretische Physik der Universität Heidelberg, Heidelberg, Federal Republic of Germany \\ and Max-Planck-Institut für Kernphysik Heidelberg, Federal Republic of Germany
}

(Received 1 December 1986)

\begin{abstract}
The production of photons from heavy-ion collisions at intermediate energies is studied in the model based on the Boltzmann-Uehling-Uhlenbeck equation. Taking into account photon production from nucleon-nucleon collisions, the theoretical results are in reasonable agreement with the experimental data at $40 \mathrm{MeV} /$ nucleon, but cross sections are smaller by about a factor of 5 , compared to experiment at $35 \mathrm{MeV} /$ nucleon. The results at these energies show no dependence on the nuclear equation of state.
\end{abstract}

Heavy-ion collisions at intermediate energies is an area which has not been explored extensively. Interesting physics is expected because of the interplay between the nuclear mean field and the residual nucleon-nucleon collisions. This is in contrast to heavy-ion collisions at low and high energies where the reaction dynamics is dominated by the nuclear mean field and the nucleon-nucleon collisions, respectively. A model based on the Boltzmann-Uehling-Uhlenbeck (BUU) equation which includes both the mean field and the Pauli blocking of nucleon-nucleon collisions has been introduced recently to describe heavy-ion collisions at intermediate energies. ${ }^{1,2}$ Important information about the properties of dense nuclear matter has been extracted by comparing experimental observables such as the collective flow and the pion production rate with those calculated with this model. ${ }^{3,4}$ However, these studies should be considered tentative as the relation of the equation of state to such observables is clouded by the dependence of these observables on dyproperties are as hard to calculate from first principles as is the equation of state, the problem of interpretation of the measurements demands that more observables be marshalled to the effort. Furthermore, both the collective flow and the pion production rate involve strongly interacting particles and are therefore significantly affected by the later stages of the collision, when the nuclear equation of state does not play an important role. To study the equation of state of nuclear matter which affects mainly the initial dynamics of the collision, it is desirable to use weakly interacting probes such as kaons ${ }^{5}$ and photons. ${ }^{6}$ namic properties of nuclear matter. Since these dynamic

Various models have been introduced to study photon production in heavy-ion collisions. They fall basically into the two categories of coherent ${ }^{7,8}$ and incoherent ${ }^{6,9-14}$ production. Within each category, the differences are in the description of the heavy-ion collision dynamics. As the BUU model is the best model available for describing heavy-ion collisions at intermediate energies, it is desirable to use this model to study photon production in heavy-ion collisions. This has been carried out recently by Bauer et al. ${ }^{12}$ Our studies, however, differ from theirs in the treatment of the photon production probability from the nucleon-nucleon collision. Also, we choose different experimental data for comparisons with the theoretical calculations in order to illustrate the differences that presently exist among the experimental data.

During the collision of two nuclei, photons are emitted from protons when they are deaccelerated by the mean field and undergo collisions with other nucleons. Since low-energy photon production is not sensitive to the detailed collision dynamics in the intermediate stage when the mean field is important, the contribution to photon production from the mean-field deacceleration of protons will not be significant. For high-energy photons, the main contribution is from individual nucleon-nucleon collisions unless the mean field changes significantly in a short time scale which is not expected for heavy-ion collisions at intermediate energies. This is confirmed by a recent study in Ref. 13.

Considering only photon production from nucleonnucleon collisions, the amplitude for emitting a photon with polarization $\varepsilon_{\lambda}$, wave vector $\mathbf{k}$, and frequency $\omega$ is given by ${ }^{6}$

$$
A_{\lambda}=i e \sum_{n}^{Z_{P}, Z_{T}} \sum_{t_{\alpha}} \exp \left[-i \omega t_{\alpha}+i \mathbf{k} \cdot \mathbf{r}\left(t_{\alpha}\right)\right]\left(\frac{\mathbf{v}_{n}^{(+)}\left(t_{\alpha}\right) \cdot \boldsymbol{\varepsilon}_{\lambda}}{-\omega+\mathbf{k} \cdot \mathbf{v}_{n}^{(+)}\left(t_{\alpha}\right)}-\frac{\mathbf{v}_{n}^{(-)}\left(t_{\alpha}\right) \cdot \boldsymbol{\varepsilon}_{\lambda}}{-\omega+\mathbf{k} \cdot \mathbf{v}_{n}^{(-)}\left(t_{\alpha}\right)}\right) \text {, }
$$

where $\mathbf{v}_{n}^{(-)}\left(t_{\alpha}\right)$ and $\mathbf{v}_{n}^{(+)}\left(t_{\alpha}\right)$ are velocities of the proton before and after the collision at time $t_{\alpha}$ and at position $\mathbf{r}\left(t_{\alpha}\right)$. The projectile and target proton numbers are denoted by $Z_{P}$ and $Z_{T}$, respectively. However, Eq. (1) is valid only in the low-frequency limit since for high frequencies, some terms in Eq. (1) will not contribute due to the Pauli blocking of the final nucleon states and, furthermore, each term in Eq. (1) will have to be weighted differently according to the density of 
final nucleon states. Since we are interested mainly or only in the high-energy photon production, which is more sensitive to the initial high-density dynamics of heavy-ion collisions, the interference between the amplitudes is insignificant and the photon emission probability is essentially given by the incoherent sum of the individual contribution from each collision. In this case, we can incorporate both effects to a good approximation in the calculation of the photon emission probability. First, we need to determine the momenta of both nucleons after the emission of a photon. This can be done conveniently in the Monte Carlo simulation of the nucleon-nucleon collision. Once the scattering angle after the collision is determined, then one can determine uniquely the momenta of the two nucleons from conservation laws. To take into account the final state density, we evaluate the phase space integral over the final state and obtain the following factor:

$$
F=\frac{m p_{3}^{3} \bar{s}}{\left|\mathbf{p}_{1}-\mathbf{p}_{2}\right| E_{1} E_{2}\left|p^{2} E_{4}-E_{3} \mathbf{p}_{3} \cdot \mathbf{p}_{4}\right|}
$$

In the above, $\mathbf{p}_{1}$ and $E_{1}$ are, respectively, the momentum and energy of the proton of mass $m$ before the collision with another nucleon with momentum $\mathbf{p}_{2}$ and energy $E_{2}$. After emitting a photon, its momentum and energy are $\mathbf{p}_{3}$ and $E_{3}$ while the other nucleon has momentum $\mathbf{p}_{4}$ and energy $E_{4}$. The quantity $\bar{s}$ is the square of the average of the initial and final center-of-mass energy of the two colliding nuclei. The phase space effect is then incorporated into the calculation by multiplying by $F$. The Pauli-blocking effect is included by introducing the factor

$$
\left[1-f\left(\mathbf{r}_{3}, \mathbf{p}_{3}\right)\right]\left[1-f\left(\mathbf{r}_{4}, \mathbf{p}_{4}\right)\right],
$$

where the $f$ 's are the phase space distribution of the nucleons after photon emission.

To determine photon production rate from heavy-ion collisions, we solve the BUU model for the collision dynamics and calculate the photon production probability perturbatively from the nucleon-nucleon collision. This is a valid approximation since the photon production cross section is very small compared with the nucleon-nucleon total cross section.

We have performed calculations using the BUU model for the reaction ${ }^{14} \mathrm{~N}+{ }^{208} \mathrm{~Pb}$ at $40 \mathrm{MeV} /$ nucleon (Ref. 15) with an isotropic nucleon-nucleon cross section of $40 \mathrm{mb}$. In Fig. 1, the photon energy spectrum at $\theta_{\mathrm{lab}}=30^{\circ}$ is shown by the solid curve which is in reasonable agreement with the experimental data. Also shown in the figure by the long-dashed curve is the contribution from nucleons in their first collisions with other nucleons. This is about one-third of the total photon yield and is consistent with the calculation by Nakayama and Bertsch ${ }^{13}$ which is given by the dashed curve. Our results from the first collision model are slightly different from theirs as we treat the distortion of the Fermi spheres more realistically. The angular distribution for $30-\mathrm{MeV}$ photon emission is shown in Fig. 2. The solid curve is the calculated results and is less forward peaked than the experimental data show.

We have also carried out calculations for the reaction of ${ }^{14} \mathrm{~N}+{ }^{58} \mathrm{Ni}$ at an incident energy of $35 \mathrm{MeV} /$ nucleon. ${ }^{16}$

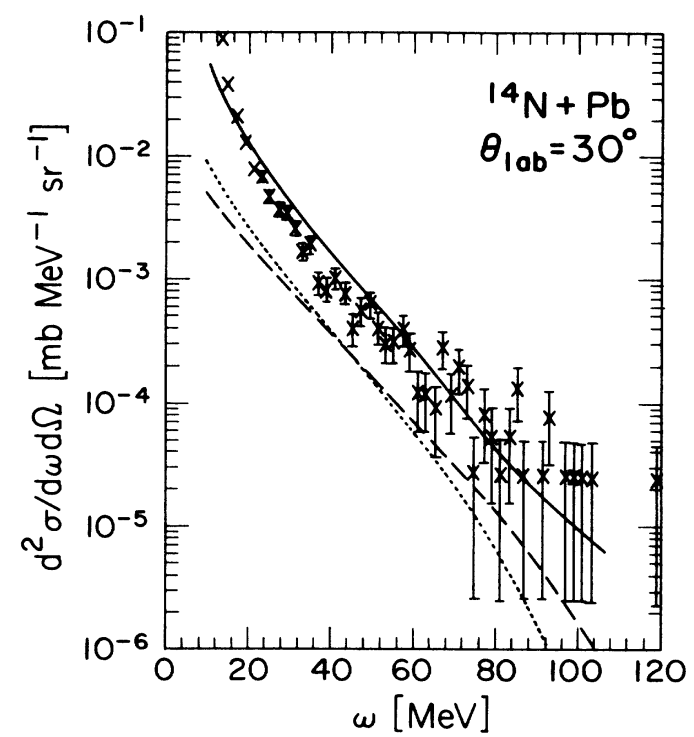

FIG. 1. Photon energy spectrum at $\theta_{\mathrm{lab}}=30^{\circ}$ for the reaction ${ }^{14} \mathrm{~N}+{ }^{208} \mathrm{~Pb}$ at an incident energy of $40 \mathrm{MeV} /$ nucleon. The solid curve is from the calculation while the data are from Ref. 15. Calculations based on the first collision model are given by the long-dashed curve from our model and by the short-dashed curve from Ref. 13.

The results are shown in Fig. 3. The theoretical results for the photon energy spectrum at $\theta_{\text {lab }}=60^{\circ}$ are given by the solid curve which is about a factor of 5 smaller than the experimental data given by the histogram. The angular distribution for photon energies between 35 and $55 \mathrm{MeV}$ is shown in Fig. 4 by the solid curve. Compared with the experimental data given by the histogram, it is seen that the theoretical angular distribution has a less prominent peak at $\theta_{\text {lab }}=60^{\circ}$.

To study the sensitivity of the photon spectra to the nuclear equation of state, we have done calculations with

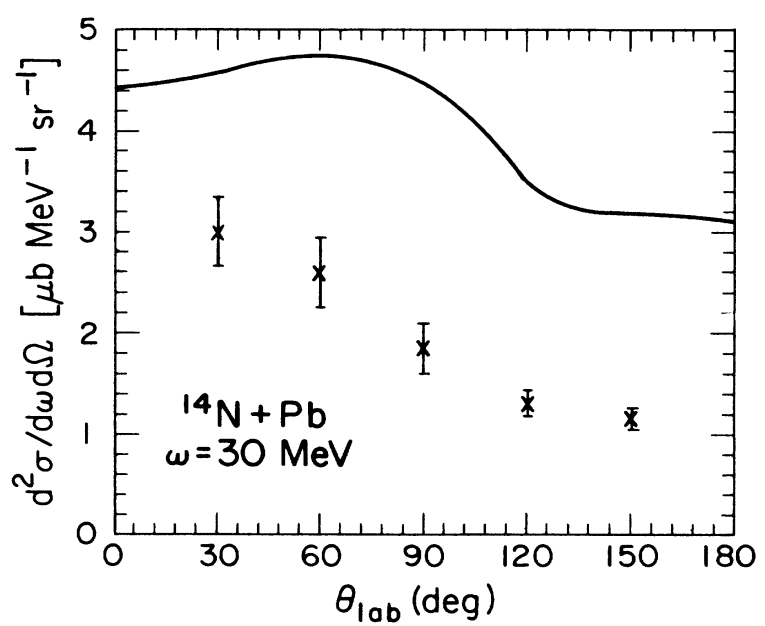

FIG. 2. Angular distribution for photon energy $\omega=30 \mathrm{MeV}$ for the same reaction as in Fig. 1. The solid curve is the calculated results while the data are from Ref. 15. 


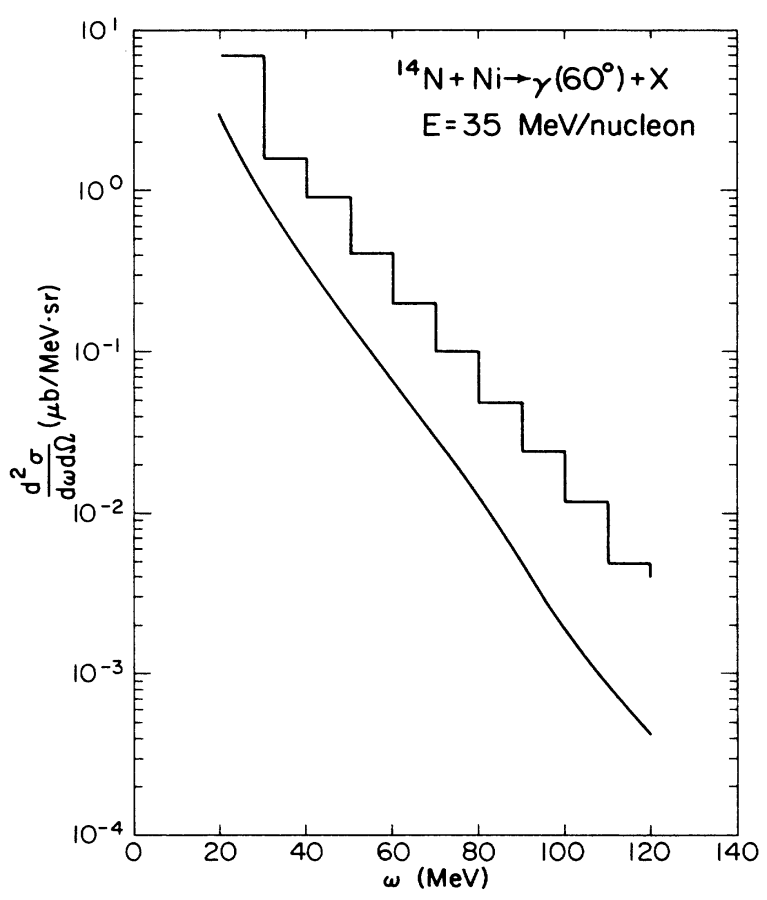

FIG. 3. Photon energy spectrum at $\theta_{\text {lab }}=60^{\circ}$ for the reaction ${ }^{14} \mathrm{~N}+{ }^{58} \mathrm{Ni}$ at an incident energy of $35 \mathrm{MeV} /$ nucleon. The solid curve is from the theoretical calculation while the histogram is the data from Ref. 16.

both a soft and a stiff equation of state, corresponding to a nuclear compressibility of 200 and $380 \mathrm{MeV}$, respectively. But calculations with the BUU model at these energies do not show a discernible dependence on the nuclear equation of state. Chiefly, this is because the maximum density reached in the central collision at such energies is only about $25 \%$ above the normal nuclear matter density. To use photons as a probe of the properties of the dense nuclear matter, it is necessary to study reactions at higher incident energies where the maximum density reached in the collision is appreciable. One then expects significant effects due to the equation of state on high-energy photon production. This is because the high-energy photons are created from nucleon-nucleon collisions at the initial stage when the nuclear density is high. Then the energy needed for compressing the nuclear matter is not available for creating particles. A soft equation of state implies that nucleons have more kinetic energies than the case of a stiff

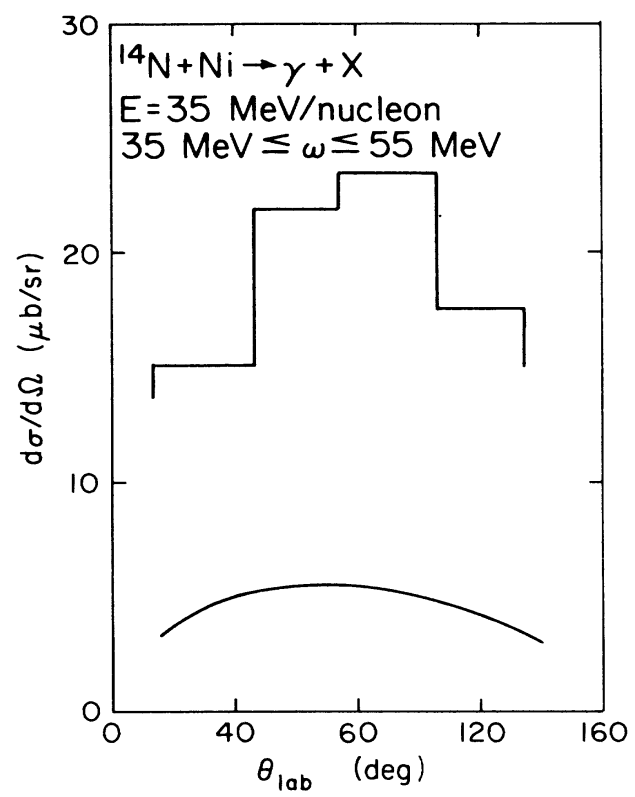

FIG. 4. Angular distribution for photon energies 35 $\mathrm{MeV} \leq \omega \leq 55 \mathrm{MeV}$ for the same reaction as in Fig. 3. The solid curve is from the theoretical calculation while the histogram is from the data of Ref. 16.

equation of state and have thus higher probabilities for producing high-energy photons. On the other hand, lowenergy photons are created whenever a proton collides with other nucleons and are thus less sensitive to the nuclear equation of state.

In conclusion, we have calculated the photon production cross section in heavy-ion collisions from the nucleonnucleon collisions only with the collision dynamics determined by the Boltzmann-Uehling-Uhlenbeck model. The calculated cross sections are in reasonable agreement with the Michigan data, but are smaller by about a factor of 5 than the Oak Ridge-Stony Brook data. We have found that the theoretical results at such low collision energies are not sensitive to the equation of state used in the BUU model. It is suggested that studies conducted at higher incident energies will have better sensitivity.

The work of one of us (C.M.K.) is supported in part by National Science Foundation Grants No. PHY-8406676 and No. PHY-8608149.
${ }^{1}$ G. F. Bertsch, H. Kruse, and S. Das Gupta, Phys. Rev. C 29, 673 (1984).

${ }^{2}$ J. Aichelin and G. F. Bertsch, Phys. Rev. C 31, 1730 (1985).

${ }^{3}$ H. Kruse, B. V. Jacak, and H. Stöcker, Phys. Rev. Lett. 54, 289 (1985).

${ }^{4}$ J. J. Molitoris and H. Stöcker, Phys. Rev. C 32, 346 (1985).

${ }^{5}$ J. Aichelin and C. M. Ko, Phys. Rev. Lett. 55, 2661 (1985).

${ }^{6}$ C. M. Ko, G. F. Bertsch, and J. Aichelin, Phys. Rev. C 31, 2324 (1985).

${ }^{7}$ D. Vasak, B. Müller, and W. Greiner, J. Phys. G 11, 1309 (1985).

${ }^{8}$ W. Bauer et al., Nucl. Phys. A456, 159 (1986).
${ }^{9}$ H. Nifenecker and J. P. Bondorf, Nucl. Phys. A442, 478 (1985).

${ }^{10}$ D. Neuhauser and S. E. Koonin, Nucl. Phys. A (to be published).

${ }^{11}$ R. Shyam and J. Knoll, Nucl. Phys. A426, 606 (1984).

${ }^{12}$ W. Bauer et al., Phys. Rev. C 34, 2127 (1986).

${ }^{13}$ K. Nakayama and G. F. Bertsch, Phys. Rev. C 34, 2190 (1986).

${ }^{14}$ B. A. Remington, M. Blann, and G. F. Bertsch, Phys. Rev. Lett. 57, 2909 (1986).

15 J. Stevenson et al., Phys. Rev. Lett. 57, 555 (1986).

${ }^{16}$ N. Alamanos et al., Phys. Lett. 173B, 392 (1986). 